\title{
Effect of freeze-thaw preparation of Ambisome on its activity against Cryptococcus gattii
}

\author{
Takashi TAMURA ${ }^{1,3,4)}$, Chihiro KIKUYAMA2), Alshahni, MOHAMED M(3)4) , Koichi MAKIMURA ${ }^{1,3,4)}$ \\ Gen. Med. Educ. Rec. Center Teikyo Univ. ${ }^{1}$, \\ Lab. Med. Mycol. Graduate Sch. Med. Teikyo Univ.2), \\ Teikyo Univ. Inst. Med. Mycol. ${ }^{3)}$, L-SEM, Graduate Sch. Teikyo Univ. ${ }^{4)}$
}

\section{【Objectives】}

The antifungal agent amphotericin $\mathrm{B}(\mathrm{AMPH}-\mathrm{B})$ is available in two forms, Fungizone that is dissolved in sodium deoxycholate and the liposomal form Ambisome(L-AMB). Both drugs have same mechanism of action, targeting fungal ergosterol. However, several studies have reported that the range of minimal inhibitory concentration (MIC) of Fungizone is $0.5-2 \mu \mathrm{g} / \mathrm{mL}$ but for Ambisome is wider with lower values, especially on Cryptococcus gattii. The aim of this study was to evaluate the effect of freeze-thaw preparation on the activity of Ambisome against $C$. gattii.

\section{【Methods】}

A total of 22 fungal strains, $C$. neoformans $(n=10)$, C. gattii $(n=12)$. Candida krusei ATCC 6258 and Candida parapsilosis ATCC 22019 were used as two quality control stains. ( Table1) MICs of Fungizone ,Ambisome and Amphotericin B prepared freshly or frozen/thawed for one round, were assessed. Both drugs were dissolved according to the manufacturer's instructions. AMPH-B were dissolved DMSO. Susceptibility tests were conducted following the M27-A3 protocol of CLSI; serial twofold dilutions were prepared with RPMI1640 medium.

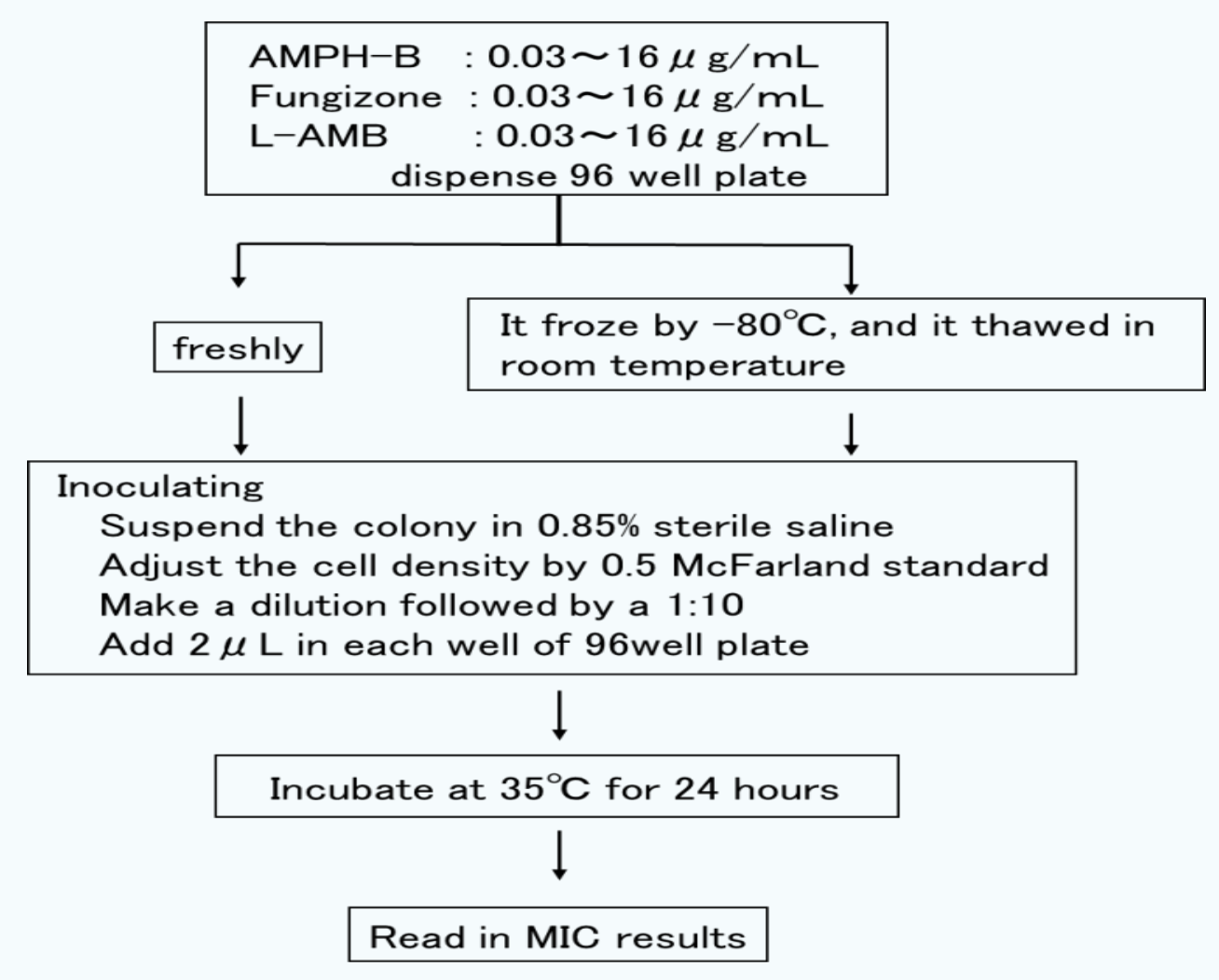

\section{【Results】}

The MIC range was $0.12-1 \mu \mathrm{g} / \mathrm{mL}$ on freshly prepared Ambisome, while it was $0.06-0.5 \mu \mathrm{g} / \mathrm{mL}$ on Ambisome with freeze/thaw preparation. Moreover, the MIC of frozen plates of Fungizone and AMPH-B was $1-4 \mu \mathrm{g} / \mathrm{mL}$. (Table 1.) A reduction of MIC of each strain by a freeze/thaw preparation was indicated below. (Fig 1 ) In addition, there was a tendency to show lower MIC values especially in $C$. gattii with a thick capsule. On the contrast, freeze/thaw preparation seems to have a less effect on strains of $C$. neoformans or $C$. gattii with a thin capsule. (Data not shown)

Table 1. Range of MIC

\begin{tabular}{lccccccc}
\hline \multirow{2}{*}{ Species } & \multirow{2}{*}{ Strains } & \multicolumn{2}{c}{ AMPH-B } & \multicolumn{2}{c}{ Fungizone } & \multicolumn{2}{c}{ Ambisome } \\
\cline { 2 - 8 } & & Fresly & freeze-thaw & Fresly & freeze-thaw & Fresly & freeze-thaw \\
\hline C. gatti sensu lato & 10 & $1 \sim 2$ & 2 & $1 \sim 2$ & $1 \sim 2$ & $0.5 \sim 1$ & $0.06 \sim 0.5$ \\
C. neformans sensu lato & 12 & $1 \sim 2$ & $2 \sim 4$ & $1 \sim 2$ & $2 \sim 4$ & $0.25 \sim 1$ & $0.12 \sim 0.5$ \\
C. krusei ATCC 6258 & 1 & 4 & 4 & 2 & 4 & 2 & 2 \\
C. parapsilosis ATCC 22019 & 1 & 1 & 2 & 1 & 2 & 1 & 0.5 \\
\hline
\end{tabular}

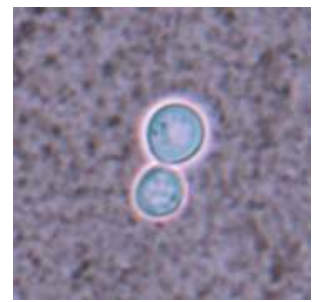

C. neoformans with a thin capsule

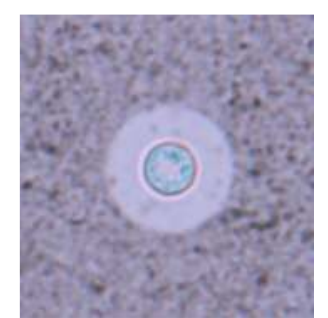

C. gattii with a thick capsule

\section{【Conclusion】}

In conclusion, it suggests that both of liposome components and capsule thickness could attributed to lower MIC values of Ambisome, as the thicker the capsule of fungi, the lower the MIC values. It assumed that the main liposome components, such as other ingredients of the liposomal medicine, phosphatidylcholinie, phosphatydyl glycerol and choresterol, could influence on the result. However its insolvability toward the culture medium makes it difficult to explore each of them in detail. In our further study, we will consider the effect of structural

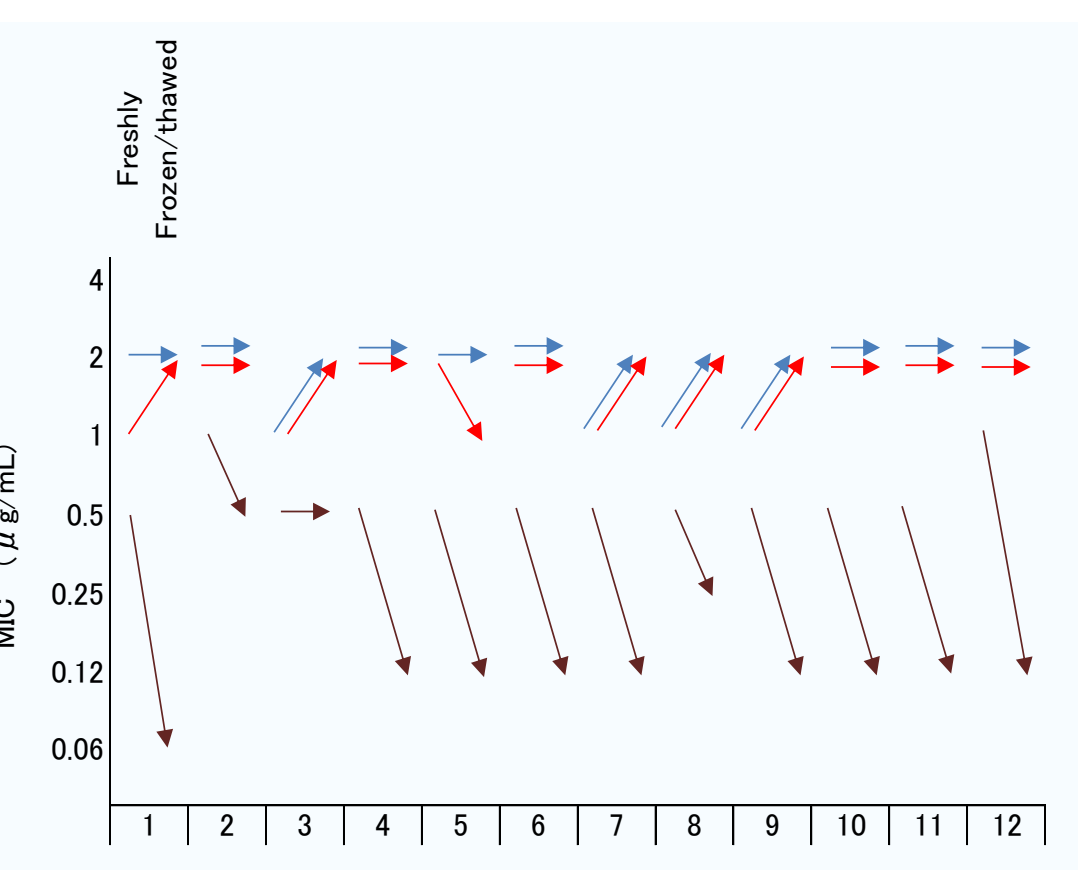

C. gatti sensu lato

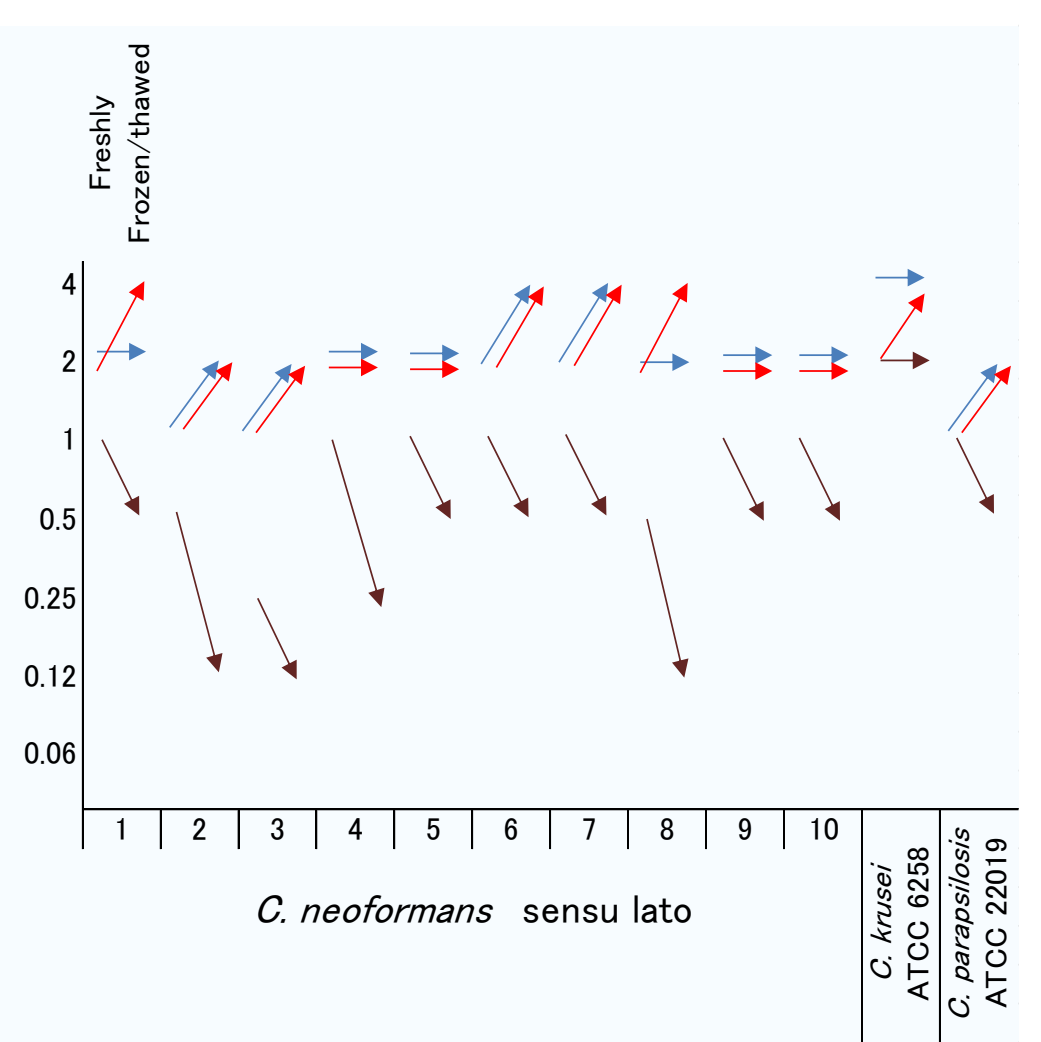

Fig 1. Effect of MIC by Freeze-thaw 